\title{
Erratum to: Two teacher quality measures and the role of context: evidence from Chile
}

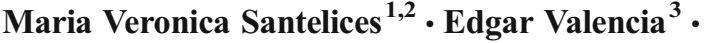 \\ Jorge Gonzalez ${ }^{4} \cdot$ Sandy $^{\text {Taut }}{ }^{5}$
}

Published online: 12 December 2016

(C) Springer Science+Business Media New York 2016

\section{Erratum to: Educ Asse Eval Acc \\ DOI 10.1007/s11092-016-9247-8}

In the original version of this article, Table 6 entries "Class", "School" and "Municipality" variables were incorrectly labeled under "Teacher fixed effects". Also, in the Appendix 1, table number was missing and the column headings were misplaced. Kindly see below correct tables. The original article was corrected.

The online version of the original article can be found at http://dx.doi.org/10.1007/s11092-016-9247-8.

Maria Veronica Santelices

vsanteli@uc.cl

Edgar Valencia

envalenc@gmail.com

Sandy Taut

staut@uc.cl

1 Facultad de Educación, Pontificia Universidad Católica de Chile, Santiago, Chile

2 Vicuña Mackenna 4860, Macul, Santiago, Chile

3 Ontario Institute for Studies in Education, University of Toronto, 252 Bloor St W, Toronto, ON M5S 1V6, Canada

4 Facultad de Matemáticas, Departamento de Estadística, Pontificia Universidad Católica de Chile, Avda. Vicuña Mackenna 4860 - Macul, Santiago, Chile

5 Escuela de Psicología, Centro de Medición MIDE UC, Pontificia Universidad Católica de Chile, Avda. Vicuña Mackenna 4860 - Macul, Santiago, Chile 
Table 6 Estimates of fixed effects in three-level model predicting NTES final category

Coefficient Standard error

Teacher fixed effects

Intercept

$1.418 \quad 0.209$

Gender

$0.053 * \quad 0.021$

Institution where teacher education was obtained

Other (Technical Institutions)

$-0.021 \quad 0.028$

Normal School

University

Type of study regimen- Full-time

$0.036 \quad 0.029$

0.000

$0.049^{*} \quad 0.018$

Continuing education (Cert., Masters, $\mathrm{PhD}$ )-Yes

$0.025 \quad 0.017$

Professional development courses-Yes

$0.043^{*} \quad 0.018$

Years of teaching experience (quadratic)

$0.000 \quad 0.000$

Grade level and subject taught

Language 8th grade

$0.029 \quad 0.030$

Math 8th grade

$0.020 \quad 0.030$

Natural sciences 8th grade

$-0.057 \quad 0.046$

Social sciences 8 th grade

$0.038 \quad 0.033$

Generalist 4th grade

$0.000 \quad$,

Expectations of maximum years of schooling of teacher's students

0.006

0.004

Intentions regarding future job prospects

Continuing as classroom teacher

$0.024 \quad 0.041$

Leaving the classroom

$0.041 \quad 0.047$

Leaving the field education

$-0.019 \quad 0.070$

Doesn't know or no response

0.000

Content coverage

$-0.018 \quad 0.022$

Perception of self-efficacy teaching a subject

$0.061 * \quad 0.028$

Teacher's classroom has a library

$\begin{array}{ll}0.016 & 0.017\end{array}$

Works in another school

$-0.005 \quad 0.026$

Holds AEP certification

$0.169 * * \quad 0.038$

Class characteristics

Number of students classroom (quadratic)

$0.000 \quad 0.000$

Class parents mean level of education

$-0.027 \quad 0.014$

Class mean family income

$0.000 * \quad 0.000$

Class parents mean expectations about maximum education level of their

$0.011 \quad 0.015$ child

Average class math score

$0.003 * \quad 0.001$

Average class language score

$-0.001 \quad 0.001$

School characteristics

Rurality (1=urban)

$0.007 \quad 0.043$

Mean school socioeconomic level

$\begin{array}{ll}-0.036 & 0.030\end{array}$

School's technology resources

$0.000 \quad 0.001$

School's pedagogical resources

$0.000 \quad 0.000$ 
Table 6 (continued)

Coefficient Standard error

\begin{tabular}{lll}
\hline Number of teachers holding certification of excellence in school & & \\
1 AEP & $0.046^{*}$ & 0.022 \\
2 AEP & 0.051 & 0.036 \\
3 o más AEP & 0.063 & 0.049 \\
No AEP & 0.000 &, \\
School average language score & $0.005^{*}$ & 0.002 \\
School average math score & -0.003 & 0.002 \\
Municipality characteristics & & \\
Type of educational administration & -0.025 & 0.031 \\
Number of students enrolled at beginning of school year & 0.000 & 0.001 \\
Percentage of people living in poverty & 0.002 & 0.002 \\
Municipal contribution over total educational income of municipality & 0.001 & 0.001 \\
\hline
\end{tabular}

$* p<0.05 ; * * p<0.01$ 


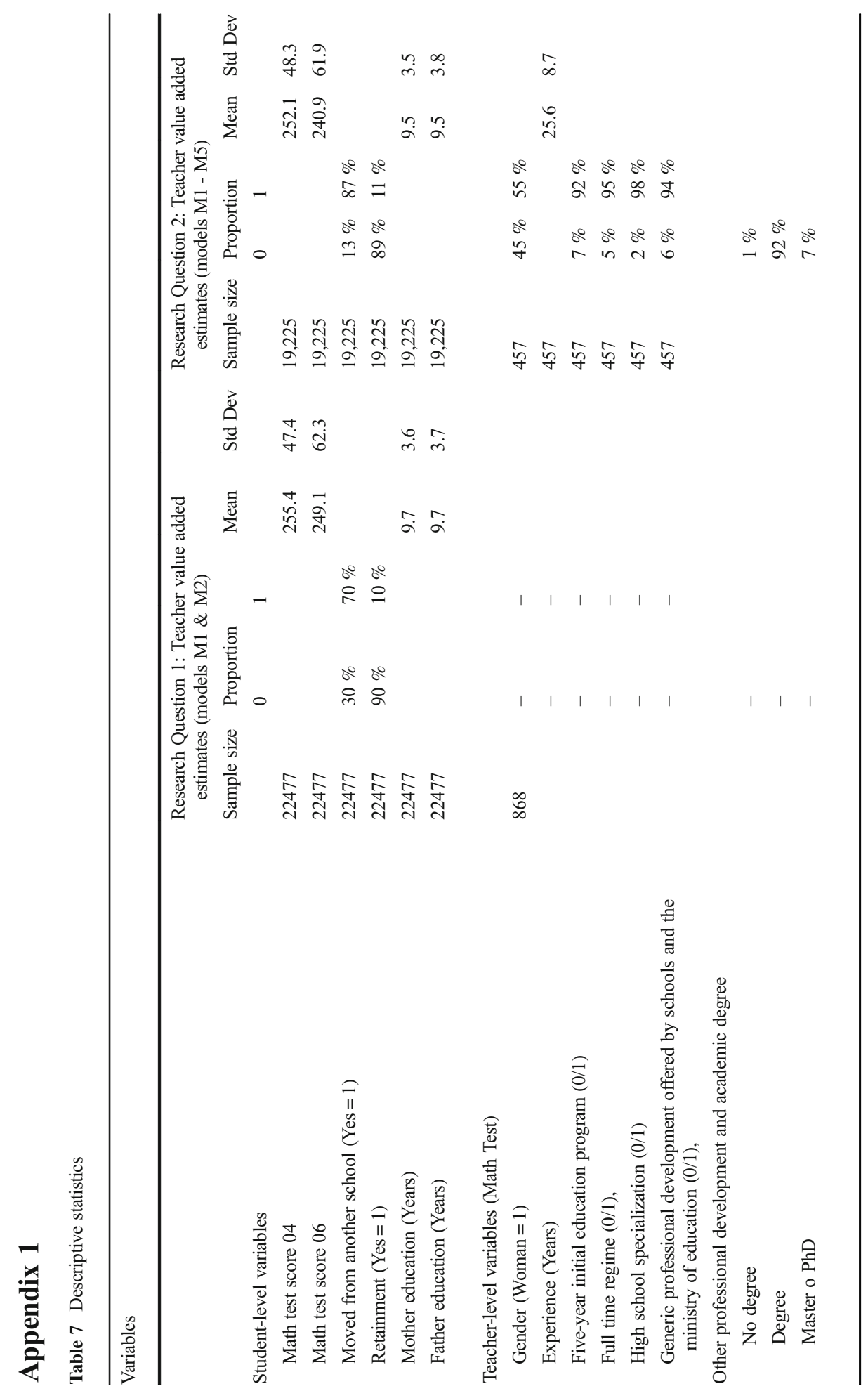




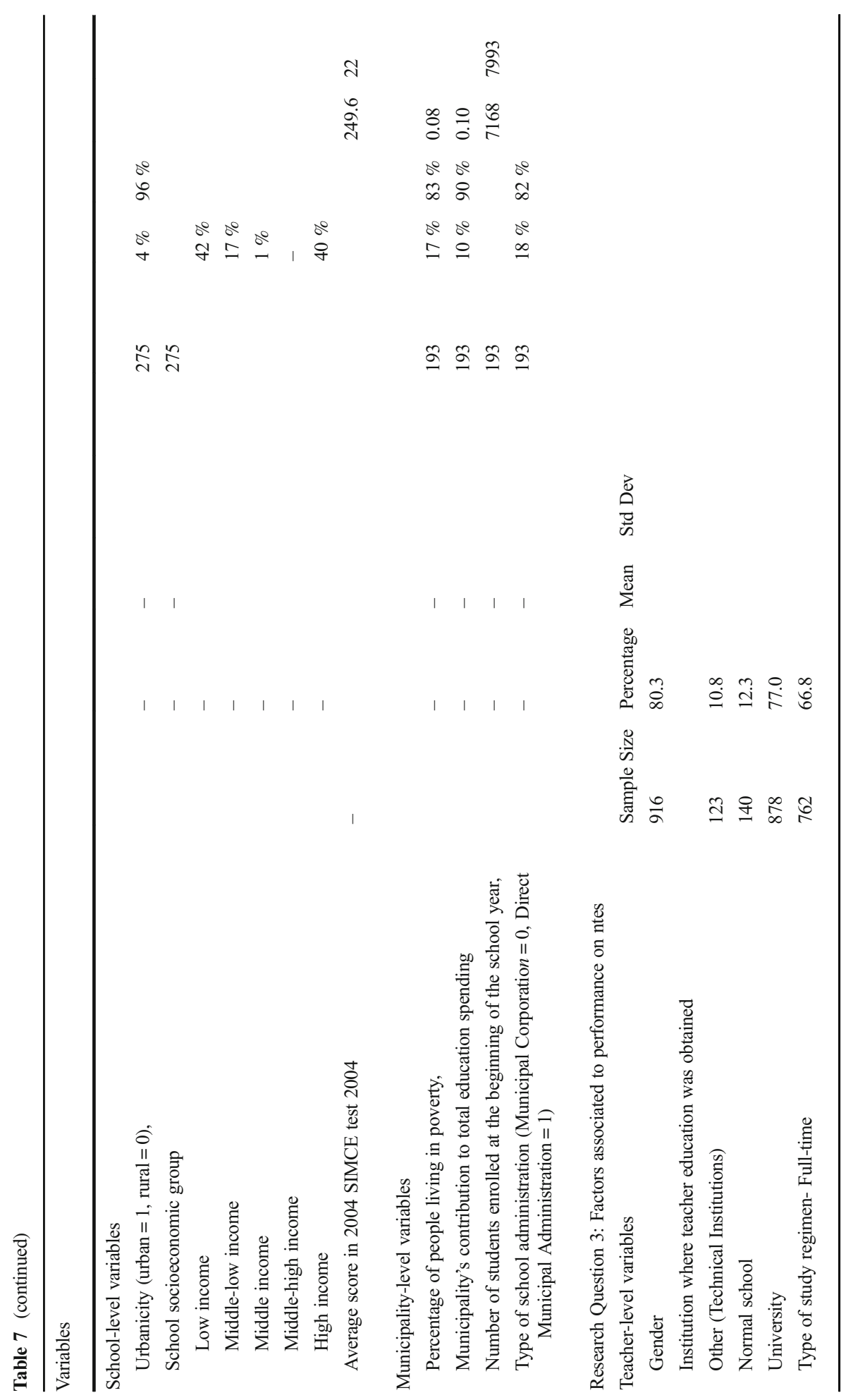




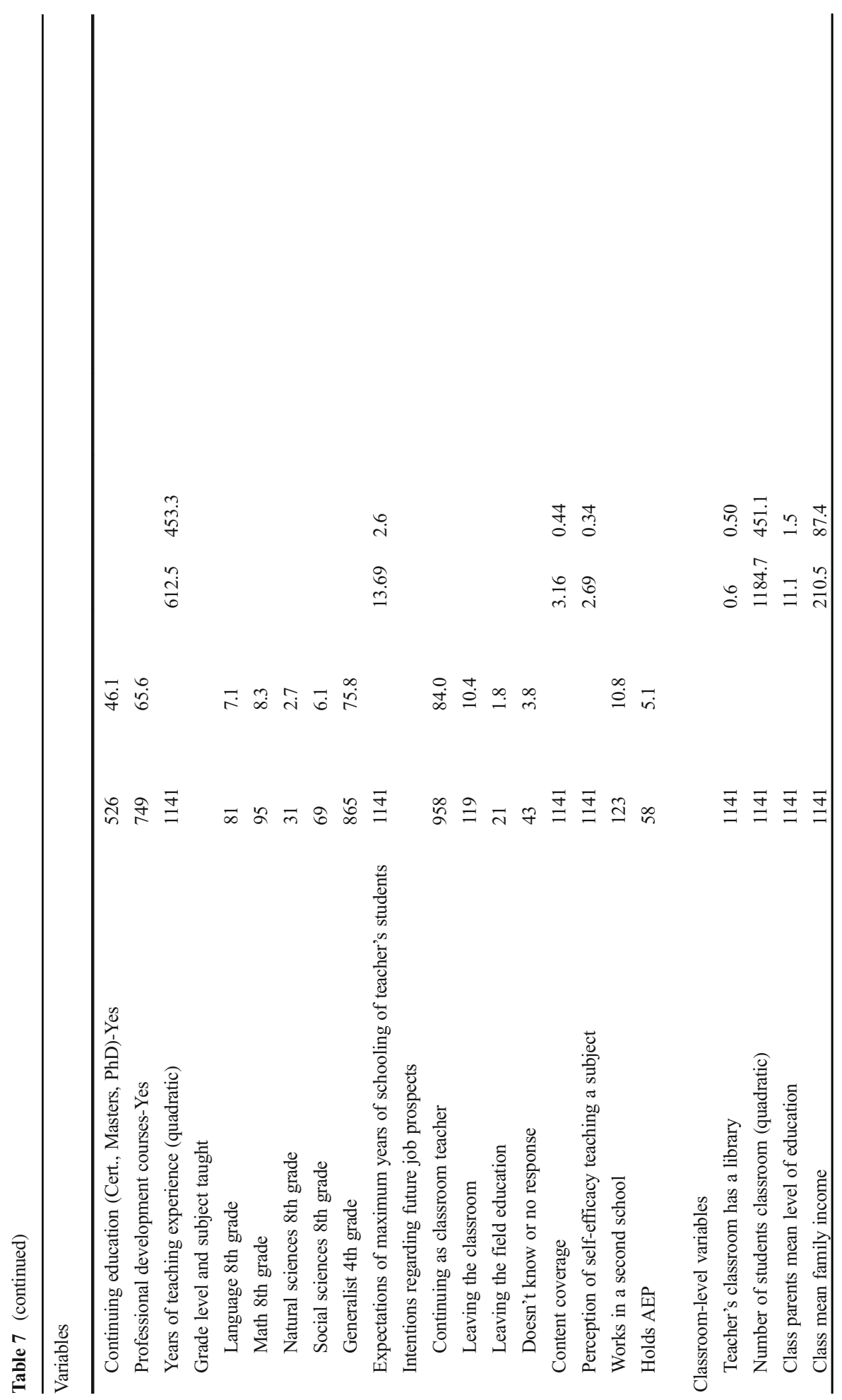




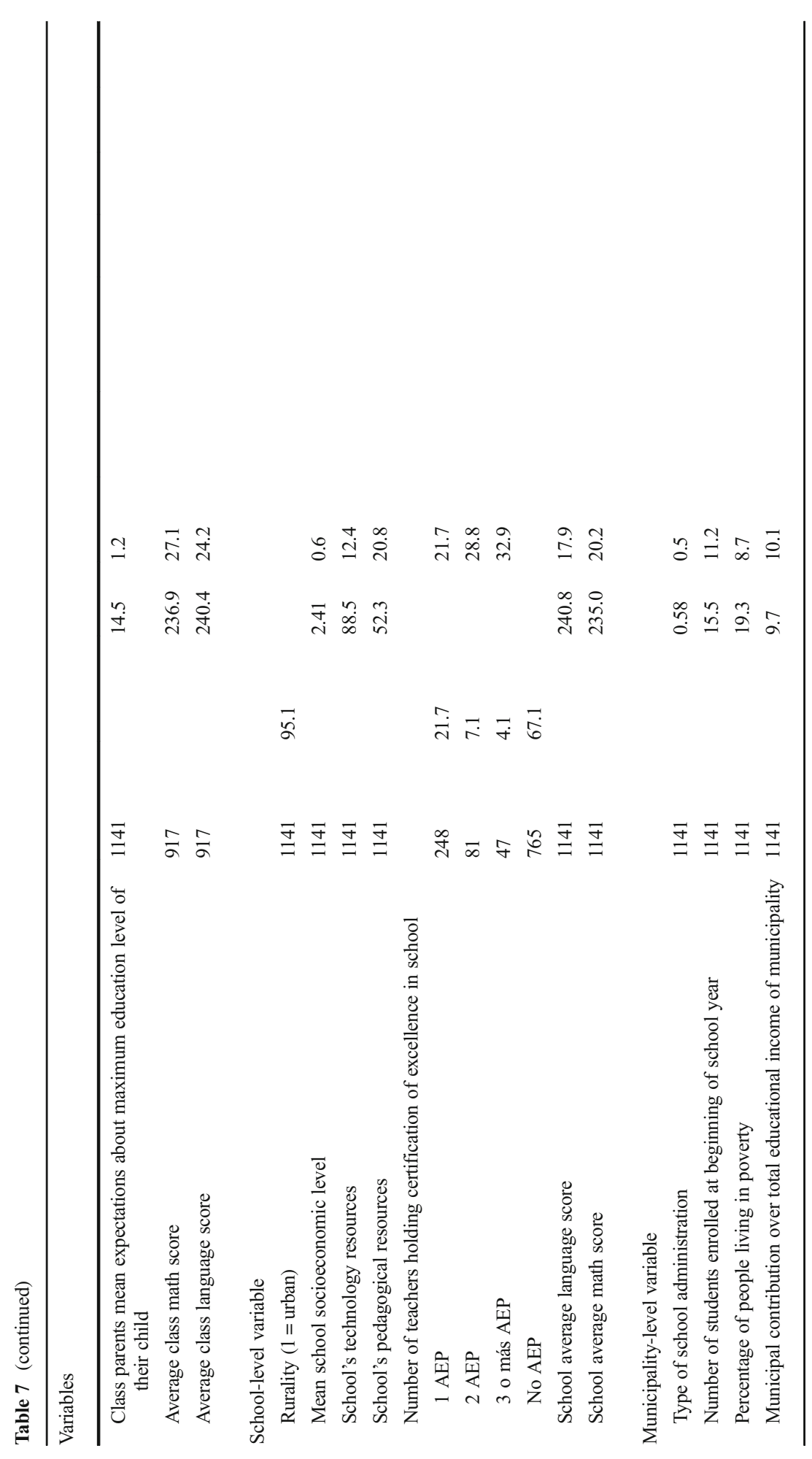

